\title{
Structured Input Activities Como Medio Para Desarrollar Estrategias de Atención a la Forma (Focus on Form) en un Contexto de Aprendizaje de Alemán Basado en Tareas
}

\author{
GLoria Bosch RoIG \\ Universidad de las Islas Baleares
}

Recibido: 24 Abril 2016/ Aceptado: 22 Mayo 2017

ISSN: $1698-7467$

\begin{abstract}
Task Based Language Learning (TBLL) can reconcile two traditional approaches effectively, on the one hand, the traditional communicative method, in which the content and the meaning are central and, secondly, the method of grammar-translation, in which the attention to the forms (focus on forms), the linguistic structure is essential. Cognitive-interactionist approaches opposed the concept of attention to the form (FoF) to the previous focus on forms $(\mathrm{FoFs})$ as focus on form $(\mathrm{FoF})$ allows direct students' attention to formal aspects of language mainly in a meaningful context of learning in a justified and reasoned manner. Selective attention can promote cognitive processes such as noticing and conscious development of form-meaning structures. How structured input activities contribute to these processes and can be applied in German task-based classes is the main objective of our analysis.
\end{abstract}

Keywords: Task Based Language Learning, Structured Input Activities, Focus on Forms, Focus on Form, Noticing.

Structured Input Activities Como Medio Para Desarrollar Estrategias de Atención a la Forma (Focus on Form) en un Contexto de Aprendizaje de Alemán Basado en Tareas

Resumen: El Aprendizaje Basado en Tareas (ABT) en las clases de lengua extranjera permite reconciliar dos enfoques tradicionales de forma eficaz, por una parte, el tradicional método comunicativo o Natural Approach, en el que los contenidos y el significado de los mismos son centrales y, por otra, métodos más estructuralistas como el audio-lingual o el de la gramática-traducción, en el que la atención a las formas lingüísticas (focus on forms), es decir, a la estructura gramatical, es primordial. Los enfoques cognitivo-interaccionistas contraponen el concepto de atención a la forma (FoF) al anterior focus on forms (FoFs), ya que focus on form (FoF) permite dirigir la atención de los alumnos hacia aspectos formales de la lengua en un contexto principalmente significativo de aprendizaje de una manera puntual, transitoria, justificada y motivada.

La atención selectiva a la forma puede favorecer procesos cognitivos como el noticing y la elaboración consciente de estructuras de forma-significado/función, en definitiva, contribuye a un aprendizaje más efectivo. De qué manera las actividades de input estructuradas (structured input activities) pueden contribuir a estos procesos y aplicarse en la clase de alemán basada en tareas, es el principal objetivo de nuestro análisis.

Palabras clave: Aprendizaje Basado en Tareas, Structured Input Activities, Focus on Forms, Focus on Form, Noticing. 


\section{INTRODUCCIÓN}

El Aprendizaje Basado en Tareas (ABT) es un enfoque cognitivo-interaccionista que parte de la premisa de que el aprendizaje o adquisición ${ }^{11}$ de una segunda o lengua extranjera (L2) se produce a través de la interacción. Philp \& Tognini (2009) definen el término "interacción" como el uso que se hace de la lengua con un fin comunicativo y un foco principalmente significativo (Focus on Meaning). Ellis (1999a, 1999b), por su parte, diferencia entre interacción interpersonal e intrapersonal. La primera se refiere a una interacción face-to-face, mientras que la segunda hace referencia a los procesos mentales internos de cada individuo que pueden ser activados y favorecidos por la comunicación interpersonal. Es importante tener en cuenta la dimensión cognitiva al hablar de la misma, ya que, como afirma Eckerth (2003), ésta representa una relación recíproca entre la acción comunicativa y los procesos de percepción y elaboración o procesamiento de la lengua. En este sentido podemos concluir que la interacción pone en marcha procesos cognitivos que pueden favorecer el aprendizaje de una L2.

De acuerdo con Rotter (2015) el Aprendizaje Basado en Tareas pone precisamente el foco en la intercomunicación, principalmente en la interacción oral, aunque no exclusivamente, ya que las tareas pueden tener como objetivo desarrollar otro tipo de destrezas, siempre que el alumno reciba suficiente input, se vea implicado en la resolución de la tarea y participe en una situación de intercambio. Para entender el ABT debemos definir primero el concepto de "tarea" y aclarar que nos referimos a actividades comunicativas que persiguen un objetivo determinado.

"[...] an activity which requires learners to use language, with emphasis on meaning, to attain an objective" (Skehan, 2003:3).

El ABT propone actividades en las cuales los estudiantes practican una comunicación significativa y pragmática para resolver tareas o realizar proyectos (Brown, 2007; Ellis, 2012; Long, 2015).

A modo de síntesis podemos afirmar que las tareas contienen tres elementos característicos fundamentales: 1. necesitan ser resueltas a través del lenguaje y la intercomunicación, 2. tienen un fin pragmático, es decir, han de alcanzar un objetivo concreto y 3. deben tener una cierta autenticidad, lo cual significa que la tarea debe tener relevancia en el mundo real, aunque no necesariamente ser auténticas, tal como apunta Skehan:

A task is an "activity in which meaning is primary, there is some sort of relationship to the real world, task completion has some priority, and the assessment of task performance is in terms of task outcome" (Skehan, 1996:38).

El ABT resulta especialmente indicado para desarrollar la competencia comunicativa en alemán de los alumnos que cursan los estudios de Turismo (Bosch, 2012, 2015), ya

\footnotetext{
${ }^{11}$ Delimitar claramente lo que es aprendizaje y adquisición de una L2 no siempre es posible, a modo orientativo definimos el aprendizaje como un proceso cognitivo más explícito, mientras que la adquisición se refiere a procesos de aprendizaje más implícitos. El aprendizaje explícito puede darse, no obstante, en situaciones de adquisición al igual que el aprendizaje implícito puede tener lugar en contextos formales. Entendiendo estos matices utilizaremos en este trabajo el término "aprendizaje", ya que se trata de un contexto formativo de alemán para turismo.
} 
que el sector demanda cada vez más una mayor competencia a los futuros profesionales debido a la creciente especialización e internacionalización de los puestos de trabajo y al continuo aumento de las exigencias respecto a los estándares de calidad. Por todo ello, los estudiantes deben ser capaces de resolver comunicativa- y eficazmente tareas concretas de su entorno profesional.

El gran potencial que ofrece el ABT para el aprendizaje de una L2, especialmente del alemán, se basa en la posibilidad de combinar el aprendizaje implícito a través de un input significativo con el aprendizaje explícito (Processing Instruction), este último a través de la aplicación de actividades y el desarrollo de estrategias de atención a la forma, teniendo siempre en cuenta que el contenido prima siempre sobre la estructura.

“[...] implicit and explicit modes of operation interact in interesting ways...[and] demonstrate that a blend of explicit instruction and implicit learning can be superior to either just explicit instruction or implicit learning alone" (Ellis, 1995:136).

A continuación vamos a ocuparnos de las conexiones de forma-función en el input a través del modelo de procesamiento (Input Processing) de VanPatten y seguidamente nos ocuparemos del aprendizaje explícito de la gramática o enfoque de atención a la forma (FOF) entendido como Processing Instruction (PI), el cual se basa en el modelo anterior.

\section{Input Processing vs. Processing Instruction}

\subsection{Input Processing}

Antes de empezar con el análisis y el diseño de las actividades estructuradas de input es necesario conocer algunos conceptos previos y entender que el aprendizaje/adquisición de una L2 no puede concebirse como un único proceso complejo, sino que incluye múltiples procesos y subprocesos que actúan en cada uno de los niveles del aprendizaje (VanPatten, 2010). En uno de estos procesos iniciales del aprendizaje los alumnos realizan conexiones entre formas gramaticales y su significado. Este proceso es denominado Input Processing (Chaudron, 1985) y tiene lugar a tiempo real en la memoria operativa: "processing is about making form-meaning/function connections during real time comprehension. It is an on-line phenomenon that takes place in working memory" (VanPatten, 2010: 7).

El modelo de procesamiento del input propuesto por VanPatten $(1993,1996,2002)$ parte de una serie de premisas. La primera (1) es que durante la interacción los alumnos están concentrados en extraer información, significado del input (Faerch \& Kasper, 1986; Krashen, 1982), la segunda afirmación (2) es que los alumnos deben darse cuenta (notice) de los elementos nuevos del input para que se produzca el aprendizaje (Schmidt, 1990). Por último (3) el noticing está limitado por la memoria operativa y su capacidad de retener y procesar la información a tiempo real (Just \& Carpenter, 1992).

A partir de las premisas anteriores, VanPatten (2010:14) establece una serie de principios y subprincipios básicos implicados en el procesamiento del input y que tienen como elemento común la primacía del significado sobre la forma: 
Principle 1. The Primacy of Meaning Principle. Learners process input for meaning before they process it for form.

Principle 1a. The Primacy of Content Words Principle. Learners process content words in the input before anything else.

Principle 1b. The Lexical Preference Principle. Learners will tend to rely on lexical items as opposed to grammatical form to get meaning when both encode the same semantic information.

Principle 1c. The Preference for Nonredundancy Principle. Learners are more likely to process non redundant meaningful grammatical form before they process redundant meaningful forms.

Principle 1d. The Meaning-Before-Nonmeaning Principle. Learners are more likely to process meaningful grammatical forms before nonmeaningful forms irrespective of redundancy.

Principle 1e. The Availavility of Resources Principle. For learners to process either redundant meaningful grammatical forms or nonmeaningful forms, the processing of overall sentential meaning must not drain available processing resources.

Principle 1f. The Sentence Location Principle. Learners tend to process items in sentence initial position before those in final position and those in medial position.

Principle 2. The First Noun Principle. Learners tend to process the first noun or pronoun they encounter in a sentence as the subject/agent.

Principle 2a. The Lexical Semantics Principle. Learners may rely on lexical semantics, where possible, instead of word order to interpret sentences.

Principle 2b. The Event Probabilities Principle. Learners may rely on event probabilities, where possible, instead of word order to interpret sentences.

Principle2c. The Contextual Constraint Principle. Learners may rely less on the First Noun Principle if preceding context constrains the possible interpretation of a clause or sentence.

Seguidamente vamos a ver de qué manera los principios básicos del modelo de procesamiento del input se integran y sirven de base para desarrollar un enfoque metodológico para la enseñanza de la gramática.

\subsection{Processing Instruction (PI)}

De acuerdo con Wong, PI es un tipo de instrucción o enfoque de atención a la forma $(\mathrm{FoF})^{12}$ basado en el modelo del Input Processing de VanPatten: "Processing Instruction (PI) is a type of focus on form instruction that is predicated or a model of input processing" (Wong, 2010: 33).

\footnotetext{
${ }^{12}$ El concepto de focus on form (FOF) contrapuesto a focus on forms (FOFs) fue desarrollado por Long (1991) y con él se pretendía diferenciar el aprendizaje explícito de la gramática practicado por los métodos tradicionales de enseñanza (FOFs) de un aprendizaje más implícito de las estructuras lingüísticas en un contexto de aprendizaje comunicativo y principalmente significativo.
} 
Gloria Bosch Roig Structured Input Activities Como Medio Para Desarrollar Estrategias de...

El objetivo de este enfoque es que los alumnos de una L2 sean capaces de procesar el input de manera más eficiente y transformarlo en intake. Hay que distinguir tres elementos principales que deben darse en este enfoque: (1) aportar información explícita sobre una determinada estructura o forma lingüística, (2) aportar información explícita sobre estrategias de procesamiento, y (3) desarrollar actividades estructuradas de input (Structured Input Strategies) (Lee \& VanPatten, 1995; VanPatten, 1993, 1996, 2000, 2002).

A diferencia de otras técnicas de atención a la forma, PI trata de identificar las estrategias erróneas o ineficientes que impiden a los alumnos procesar correctamente una determinada forma lingüística. Una vez han sido identificadas estas estrategias, teniendo en cuenta los principios formulados en el apartado anterior, el siguiente paso consistirá en desarrollar una serie de actividades de atención a la forma que ayuden a los alumnos a procesar el input más eficientemente.

Podemos afirmar, por tanto, que PI es un instrumento pedagógico o metodológico, un enfoque basado en el Input Processing, siendo éste último un modelo de análisis del input para realizar conexiones de forma-significado/función (Wong, 2010: 35).

El siguiente apartado vamos a dedicarlo a las actividades estructuradas de input, ya que pueden convertirse en una herramienta didáctica muy útil para promover la atención a la forma en contextos de aprendizaje significativos como el ABT.

\subsubsection{Structured Input Activities}

La función didáctica o pedagógica de este tipo de actividades consiste en modificar o manipular el input para que los alumnos presten más atención a las conexiones de forma-significado/función. No se trata de que los estudiantes produzcan la estructura, sino de que a partir de la forma o estructura lingüística presentada, interpreten su significado o función. Veamos cuáles son las principales características de las actividades estructuradas de input.

Una premisa distintiva y fundamental para poder hablar de actividades estructuradas de input es que dichas actividades se deriven de un análisis previo de los problemas de procesamiento que los alumnos hayan podido tener con una determinada forma o estructura lingüística. Ante problemas de procesamiento de los alumnos, la pregunta que debemos formularnos es qué estrategias erróneas han utilizado, aplicando para ello los principios y subprincipios propuestos por VanPatten (ver 2.1.).

De acuerdo con Lee \& VanPatten (1995), VanPatten (1996) y Wong (2010:38), para desarrollar las actividades de input estructuradas es necesario seguir las siguientes directrices:

1. Present one thing at a time. Es recomendable poner solamente una forma o función en el foco de atención de los alumnos, ya que éstos tienen una capacidad limitada para procesar la información. Es más fácil prestar atención cuando hay menos elementos a lo que prestar atención.

2. Keep meaning in focus. Es necesario en todo momento primar el significado sobre la forma lingüística. No existe aprendizaje sin exposición a un input significativo, por ello el procesamiento de la forma o estructura durante la actividad no puede llevarse a cabo sin atender al input significativo.

3. Move from sentences to connected discourse. Procesar frases cortas es mucho más fácil que hacerlo con textos o frases largas y los alumnos prestarán más atención a los elementos lingüísticos nuevos. 
Integrar secuencias de atención a la forma como las actividades estructuradas de input en un contexto comunicativo de aprendizaje del alemán basado en tareas puede resultar especialmente beneficioso para los alumnos, ya que con ello podemos atraer su atención hacia estructuras gramaticales o aspectos más formales de la L2, en algunos casos más marcados respecto a la L1, en nuestro caso el español, aunque también por ello menos salientes y perceptibles para ellos, y como consecuencia, más difíciles de aprender implícitamente. Un buen ejemplo de ello es la flexión nominal, pronominal y adjetival, especialmente los morfemas flexivos en alemán.

Otra ventaja de integrar este tipo de actividades en un contexto ABT es que podemos pasar del procesamiento del input a la producción u output, y con ello también podemos validar las actividades de atención a la forma.

Sin embargo, cabe preguntarse si este tipo de actividades es aplicable e igualmente eficiente a la hora de tratar cualquier dificultad respecto la gramática.

\section{Estrategias aplicadas por los alumnos de alemán para la resolu- CIÓN DE UNA TAREA ESCRITA}

A continuación, vamos a analizar los resultados de una tarea realizada por estudiantes de Alemán para Turismo con el objetivo de detectar estrategias de procesamiento ineficientes y hacer un diagnóstico de las mismas. Seguidamente propondremos un diseño de tareas estructuradas y actividades de atención a la forma que tengan como objetivo el desarrollo de estrategias más eficientes que promuevan el noticing ${ }^{13}$, así como un aprendizaje más explícito y consciente.

\subsection{Definición de la tarea y consideraciones previas}

La tarea propuesta a los alumnos consistía en responder a un correo electrónico de un amigo alemán. Se pidió a los estudiantes que en su respuesta tuvieran en cuenta una serie de preguntas relacionadas.

Antes de realizar esta tarea, los alumnos habían recibido información explícita sobre la estructura de un correo electrónico y de una carta formal o comercial (diferenciando principalmente cuatro partes: 1. Encabezamiento, 2. Introducción, 3. Tema y 4. Despedida). También habían practicado y escrito una carta formal reaccionando a una reclamación de un cliente. Para llevar a cabo la tarea escrita, los alumnos recibieron previamente instrucción explícita sobre las fórmulas de cortesía y una serie de estructuras, frases hechas y chunks ${ }^{14}$.

${ }^{13}$ Schmidt (1990) y Terrel (1991) entienden por noticing el registro consciente de una forma o estructura gramatical del input sin que se haya producido necesariamente una asignación semántica o funcional a la misma. Schmidt (2001) y VanPatten (2010) diferencian input de intake, ya que éste último término hace referencia al material lingüístico ya procesado en el que se ha producido la conexión forma-función de una estructura.

${ }^{14}$ Se trata de estructuras significativas base del aprendizaje, ya sean palabras, sintagmas, colocaciones o frases, que los alumnos aprenden de forma contextualizada e implícita, por imitación o fusión, sin que haya habido un análisis gramatical previo de las mismas, y que forman parte de la interlengua de los alumnos. A medida que los alumnos van avanzando en el aprendizaje de la L2, pueden ir modificando y añadiendo nuevos elementos lingüísticos a los chunks. 
Un mes después de tratar el tema de la carta comercial en clase, anunciamos a los alumnos que realizaríamos próximamente un test consistente en escribir una carta o correo electrónico. En ningún momento les revelamos si el escrito que les íbamos a pedir era de tipo personal o formal. Tanto cartas como correos personales habían sido tratados ya en cursos y sesiones anteriores.

\subsection{Input y Planteamiento de la tarea}

Sie wollen im Sommer in Deutschland einen Intensivdeutschkurs machen und brauchen eine Unterkunft für zwei Monate. Ein deutscher Freund macht Ihnen in seiner E-Mail folgenden Vorschlag:

Liebe/r....

Ich freue mich schon sehr auf unser Treffen im Sommer! Da ich nur ein kleines Zimmer in einer WG habe, kannst du leider nicht bei mir übernachten. Aber viele Studenten fahren im Sommer nach Hause und du könntest mit ein bisschen Glück ein Zimmer in einem Studentenwohnheim bekommen. Dort gibt es meistens Einzelzimmer. Wir können auch versuchen, für dich ein Zimmer in einer WG zu bekommen. In einer Wohngemeinschaft wohnen meistens 2 bis 4 Personen. Jeder hat ein eigenes Zimmer. Küche, Bad und Wohnzimmer sind für alle da. Wenn du mit Deutschen in einer WG wohnst, sprichst du mehr Deutsch und kannst deine Deutschkenntnisse schneller verbessern.

Schreib mir bitte so bald wie möglich, wann du kommen willst, wie lange du das Zimmer brauchst und wie viel Geld du dafür ausgeben kannst. Ich kümmere mich dann um das Zimmer und schreibe dir, was ich gefunden habe.

Viele Grüße

Andreas

Beantworten Sie Andreas E-Mail. Folgende Punkte sollten enthalten sein: wie viel Geld Sie maximal für die Miete ausgeben können, was Sie gern in Ihrer Freizeit unternehmen wollen, wann Sie nach Deutschland kommen, bis wann Sie bleiben und wo Sie am liebsten wohnen wollen.

Texto extraído del libro Studio D B1 ed. Cornelsen (2007)

\subsection{Resultados}

En este apartado hemos analizado los E-Mails de los 40 alumnos participantes en la tarea y nos hemos centrado fundamentalmente en tres de las cuatro partes en que hemos estructurado el escrito: el encabezamiento, la introducción y la despedida, ya que realizar un análisis del tema hubiera trascendido el marco de este trabajo. Hemos considerado como formas incorrectas la utilización inadecuada de una fórmula o expresión según el contexto social y la intención comunicativa. Los errores incluyen la ortografía y todos los aspectos gramaticales. 
Tabla 1. Total de formas incorrectas o formas que presentan errores

\begin{tabular}{|c|c|c|c|}
\hline N=40 & Encabezamiento & Introducción & Despedida \\
\hline Total F.I. & $15 / 37.5 \%$ & $31 / 77.5 \%$ & $\mathbf{2 8} / \mathbf{7 0} \%$ \\
\hline
\end{tabular}

\subsubsection{Encabezamiento}

En el encabezamiento es donde menos errores se observan. La fórmula que hemos considerado como correcta para esta tarea era "Lieber Andreas" o también "Hallo Andreas". 25 alumnos han elegido la fórmula correcta y 15 alumnos han cometido errores en el encabezamiento. El error que se repite más veces (12) es "Liebe Andreas". También aparecen las fórmulas incorrectas "sehr geehrte Andreas", "sehr geehrte Herr Andreas" y "seehr geehrte Andreas".

Podemos inferir de los errores en el encabezamiento que ha habido problemas en diferenciar fórmulas de saludo formales (sehr geehrter/e) e informales (Lieber/e) que afectan al plano semántico y pragmático (Herr Andreas). Por otro lado, estas fórmulas presentan errores que afectan tanto a la ortografía (seehr) como a la flexión adjetival (*Liebe Andreas, *sehr geehrte Andreas). En este último caso los alumnos utilizan la forma menos marcada, menos compleja "liebe" en lugar de "lieber", que es redundante, ya que el sustantivo "Andreas" contiene toda la información y marca también el género; lo mismo ocurre con "**sehr geehrte Andreas o *sehr geehrte Herr Andreas". Se cumple aquí el principio de no redundancia (1c), los alumnos prefieren procesar formas no redundantes que sean significativas en lugar de formas redundantes portadoras de significado.

\subsubsection{Introducción}

En la introducción es donde más errores se han constatado. Tan sólo 6 alumnos han elegido y utilizado fórmulas correctamente, 3 alumnos no han escrito ninguna introducción, han empezado directamente con el asunto y 31 han cometido errores. Los errores muestran una gran variabilidad:

*Danke für ihre Brief

*vielen Dank für Ihnen Schreiben

*vielen dank fürh ihre Briefe

*ich freue mich schon auf deinen Brief

*ich freue mich schön über deinen Brief

*vielen Dank für deine Brief

*vielen Dank für ihrem Brief, mit dem ich sehr erfreut bin

*vielen Dank für Ihre wichtigen Information

*ich danke sehr dir, dass du mit meine Wohnung mir hilfst

*Danke für Ihrem Brief

*vielen Dank für die Brief

*viele Dank für ihre antworten

*viele Danke für Dein Schreiben

*vielen Dank für Ihne Brief 
Gloria Bosch Roig Structured Input Activities Como Medio Para Desarrollar Estrategias de...

*vielen dank für deinen Brief und ich habe Lust dir zu sehen

*Ich bin sehr zufrieden auch auf unser Treffen für zwei Monaten

*vielen Dank für Ihre Hilfe

*vielen Dank für alle Information

*vielen Dank für dein Brief und deine Lust mir zu helfen, und entschuldige mich für die spätteste Antwort.

En esta parte observamos los mismos problemas de procesamiento que en el encabezamiento, aunque se agravan los relacionados con la flexión, especialmente la pronominal en sintagmas preposicionales: *für dein Brief, *für Ihne Brief, *für ihrem Brief, *für ihre Brief, * für Ihnen Schreiben. Se cumplen aquí las predicciones del principio $1 c$ de no redundancia. Los alumnos procesan primero "für" y "Brief" como elementos portadores de más significado "für" como preposición de acusativo prima sobre la marca de la flexión del posesivo que resulta redundante.

Lo mismo ocurre con otros morfemas flexivos: *viele Danke, *viele Dank. Aparecen también algunos pocos problemas relacionados con el orden de las palabras. Los errores en la sintaxis surgen en frases más largas y elaboradas (*ich danke sehr dir, dass du mit meine Wohnung mir hilfst, *Ich bin sehr zufrieden auch auf unser Treffen für zwei Monaten).

Vemos que se cumple aquí el principio $1 c$ respecto a la posición de los elementos de la frase, los alumnos prefieren procesar los elementos en posición inicial y final de la frase: *Ich danke sehr dir en lugar de: ich danke dir sehr/*dass du mit meine Wohnung mir hilfst en lugar de: dass du mir mit meiner Wohnung hilfst.

\subsubsection{Despedida}

En la parte final del correo, la despedida, hemos encontrado 12 formas empleadas correctamente y 28 incorrectas. La fórmula "Viele Grüße" es la más empleada, aunque no siempre sola, se repite 22 veces y esto podría sugerir que los alumnos la han importado directamente del texto de referencia.

En los errores encontramos también una gran variabilidad:

*Ich hoffe, dass wir sehr bald sehen. Viele Grüße.

*Schreibt mir sofort, bitte. Viele Grüße.

*vielen Dank und ich hoffe, sehr du wieder bei mir begrüßen zu dürfen. Viele Grüße

* Schreib mir bitte so bald. Viele Grüße.

*Mit freundliche Grüßen

*Mit freundliche Gruße

*Mit feundlichen Grußen

*Viele Gruße

*Danke schon, bis july. Viele Grüße

*Schreib mir bitte, wenn du eine Wohnung treffen. Viele Grüße

*Vielen Dank für Ihre E-mail und Ihre Hilfe. Mit freundlischen Grüßen

*Liebe Gruße. Ich hoffe, bald von Ihre zu hören

*Dank für deine Hilfe. Mit freundliche Grüße

*Danke für Ihre hilfe und auf weider schreiben. Viele Gruße 
*Ich erwarte deine nächste Brief

*Vielen dank für ihre helfen. Ich hoffe, Sie bald in Berlin.Viele Grüße

De la misma manera que en la primera y segunda parte, los alumnos emplean fórmulas propias de cartas formales en un escrito personal (*vielen Dank für Ihre E-Mail und Ihre Hilfe. Mit freundlichen Grüßen, *vielen Dank und ich hoffe, sehr du wieder bei mir begrüßen zu dürfen. Viele Grüße, *Danke für ihre helfen. Ich hoffe, Sie bald in Berlin. Viele Grüße).

También observamos en esta última parte un mayor empleo del chunking por parte de los alumnos, estructuras que han aprendido de forma holística, sin analizar, se cumple el principio $l$ y los subprincipios $l a$ y $l b$ de primar el contenido sobre la forma y de procesar preferiblemente las palabras, elementos léxico-semánticos más grandes antes que elementos más pequeños como los morfemas flexivos. Los chunks presentan mucha variabilidad interna $(*$ Liebe Gruße. Ich hoffe, bald von Ihre zu hören, *Mit freundliche Grüßen *Mit freundliche Gruße, *Mit feundlichen Grußen, *Danke für Ihre hilfe und auf weider schreiben. Viele Gruße, *Liebe Gruße).

Para diseñar actividades estructuradas de input que resulten en estrategias más eficientes según el enfoque del PI, siempre tendremos que identificar primero los problemas de procesamiento del input para redirigir la atención de los alumnos sobre estos elementos o estructuras de forma-función especialmente complejos.

\section{Propuesta de actividades estructuradas de input como Estrategias DE ATENCIÓN A LA FORMA}

Seguidamente presentamos una serie de actividades de atención a la forma en las que hemos querido promover el noticing de conexiones forma-función consideradas de difícil procesamiento según el análisis del apartado anterior, para ello hemos manipulado el input poniendo en el foco de atención dichas relaciones. En muchos casos hemos empleado la letra negrita para hacer los elementos formales más salientes y perceptibles para los alumnos. También hemos aumentado la frecuencia de aparición de muchas de ellas, integradas en frases o estructuras cortas, presentando un contexto significativo a través de los enunciados.

Es importante que los alumnos reciban un feedback explícito después de realizar los ejercicios.

4.1. Decide qué pronombres utilizamos en una carta o correo formal para dirigimos al receptor, por ejemplo, al cliente de un hotel. Márcalos con una $\mathrm{X}$

Sie ( ) Ihnen ( ) Du ( ) Ihr ( ) Ihre ( ) Dir ( ) Dein ( ) Deine ( )

4.2. Decide qué pronombres utilizamos en una carta o correo informal para dirigimos al receptor, por ejemplo, a un amigo o amiga alemán. Márcalos con una $\mathrm{X}$

Sie ( ) Ihnen ( ) du ( ) Ihr ( ) Ihre ( ) dir ( ) dein ( ) deine ( ) 
Gloria Bosch Roig Structured Input Activities Como Medio Para Desarrollar Estrategias de...

4.3. Decide qué fórmulas de encabezamiento de cartas y correos son formales (F) y cuáles informales (IF)

Liebe Ana ( ) Sehr geehrte Frau Mayo ( ) Hallo Maria ( ) Lieber Freund ( ) Liebe Schwester ( ) Lieber Vater, liebe Mutter ( ) Sehr geehrte Damen und Herren

( ) Lieber José ( ) Liebe Freunde ( ) Sehr geehrter Herr Gómez

( ) Liebe Freundin ( )

Sehr geehrter Herr Ruíz, sehr geehrte Frau Sanz ( ) Liebe Eltern ( )

Mein Lieber Andreas ( )

4.4. Decide qué fórmulas de encabezamiento NO utilizarías en una carta o e-mail a tu amigo Andreas por inadecuadas o incorrectas. Márcalas con una X. Razona tu respuesta

Liebe Andreas ( ) Sehr geehrter Andreas ( ) Hallo Andreas ( ) Lieber Freund Andreas ( ) Lieber Andreas ( ) Sehr geehrter Herr Andreas ( ) Sehr geehrte Herr Andreas ( )

Mein lieber Andreas ( )

\subsection{Completa las frases de agradecimiento formal}

Sehr geehrter Herr Suau, vielen Dank für

Sehr geehrte Frau Villa, vielen Dank für

Sehr geehrter Herr López, vielen Dank für

Sehr geehrte Frau Bosch, vielen Dank für

Sehr geehrter Herr Sánchez, vielen Dank für

Schreiben.

Sehr geehrter Herr Bernat, vielen Dank für E-Mail. Brief. Antwort Hilfe. wichigen Informationen.

\subsection{Completa las frases de agradecimiento informal}

Liebe Anna, vielen Dank für Lieber Jens, vielen Dank für Brief.

Liebe Andrea, vielen Dank für Antwort.

Lieber Freund, vielen Dank für Liebe Maya, vielen dank für Schreiben.

Lieber Martin, vielen Dank für Hilfe. in zwei Monaten

Liebe Steffi, vielen Dank für mich für die späte Antwort.

Lieber Luís, ich danke dir sehr, dass

4.7. Decide qué fórmulas de despedida en cartas y correos son formales (F) y cuáles informales (IF)

Ich hoffe, dass wir uns sehr bald sehen. Viele Grüße ( )

Schreib mir sofort, bitte. Viele Grüße ( ) 
Schreib mir bitte bald. Viele Grüße ( )

Mit freundlichen Grüßen ( )

Viele Grüße ( )

Danke schön. Viele Grüße ( )

Schreib mir bitte, wenn du eine Wohnung findest. Viele Grüße ( )

Vielen Dank für Ihre E-Mail und Ihre Hilfe. Mit freundlichen Grüßen ( )

Liebe Grüße. Ich hoffe, bald von Ihnen zu hören ( )

Danke für Ihre Hilfe. Mit freundlichen Grüßen

Ich erwarte deinen nächsten Brief ( )

Vielen Dank für Ihre Hilfe. Ich hoffe, Sie bald in Berlin zu sehen.Viele Grüße ( )

Vielen Dank und ich hoffe, Sie wieder bei mir begrüßen zu dürfen. Viele Grüße ( )

4.8. Decide qué fórmulas de despedida NO utilizarías en una carta o e-mail a tu amigo Andreas por inadecuadas o incorrectas. Márcalas con una X. Razona tu respuesta

Ich hoffe, dass wir uns sehr bald sehen. Viele Grüße ( )

Schreib mir sofort, bitte. Viele Grüße ( )

Vielen Dank und ich hoffe, sehr du wieder bei mir begrüßen zu dürfen. Viele Grüße ( )

Schreibt mir bitte so bald. Viele Grüße ( )

Mit freundliche Grüßen ( )

Mit freundliche Gruße ( )

Mit feundlichen Grußen ( )

Viele Gruße ( )

Danke schon, bis july. Viele Grüße ( )

Schreib mir bitte, wenn du eine Wohnung treffen. Viele Grüße ( )

Vielen Dank für Ihre E-mail und Ihre Hilfe. Mit freundlischen Grüßen ( )

Liebe Gruße. Ich hoffe, bald von Ihre zu hören ( )

Dank für deine Hilfe. Mit freundliche Grüße ( )

Danke für Ihre hilfe und auf weider schreiben. Viele Gruße ( )

Ich erwarte deine nächste Brief ( )

Vielen Dank für ihre helfen. Ich hoffe, Sie bald in Berlin.Viele Grüße ( )

\section{Conclusión}

Para concluir quisiera reiterar la importancia del input en contextos interactivos de enseñanza-aprendizaje como el ABT para la adquisición de una L2. Es cierto, y existe consenso sobre este aspecto entre los investigadores (Swain, 1991), que suministrar input significativo a los alumnos no es suficiente, también es necesario dirigir su atención hacia aspectos más formales de una lengua, y esto resulta imprescindible en el caso del alemán en el contexto que nos ocupa, ya que se trata de una lengua mucho más flexiva que cualquier otra lengua románica o que el inglés.

Como hemos visto en apartados anteriores, los morfemas flexivos resultan especialmente difíciles de procesar a nuestros alumnos, por ello, el aprendizaje explícito o (Processing 
Instruction) del input se hace imprescindible en todos los estadios del aprendizaje, fundamentalmente en el inicial. Las actividades estructuradas de input pueden resultar una potente herramienta para desarrollar estrategias de procesamiento más eficientes en los alumnos, sobre todo para tratar relaciones de forma-función/significado que resulten más complejas de procesar como es el caso de la flexión gramatical.

La aplicación de actividades y el desarrollo de estrategias de atención a la forma, teniendo siempre en cuenta que el contenido prima sobre la forma/estructura, puede realizarse perfectamente en contextos de enseñanza-aprendizaje basado en tareas, ya que se trabaja con input auténtico y significativo, se favorece la interacción y el output, y de esta manera podemos pasar de secuencias explícitas de atención a la forma a fases de interacción y producción, mediante las cuales podemos detectar posibles estrategias de procesamiento erróneas y también es posible evaluar el éxito o validar las actividades de atención a la forma. Adicionalmente el output ayuda a los alumnos a fijarse en la forma:

I have hypothesized that, under certain circumstances, output promotes noticing. This is important if there is a basis to the claim that noticing a form in input must occur in order for it to be acquired. (Swain, 1998:66)

\section{REFERENCIAS BIBLIOGRÁFICAS}

Bosch, G. (2012). "Aufgabenorientierung mit proaktiver Formfokussierung als didaktisches Konzept für den Deutschunterricht im Tourismus", en IBERICA, 23: 157-172.

Bosch, G. (2015). "Design und Implementierung von Formfokussierungsaufgaben im interaktiven ihnhalts- und bedeutungsorientierten DaFT-Unterricht. Eine Fallstudie im Rahmen eines Aktionsforschungsprojekts", en IBERICA, 30: 37-59.

Brown (2007). Teaching by Principles: An Interactive Approach to Language Pedagogy. Upper Saddle River, NJ: Pearson.

Chaudron, C. (1985). "Intake: On methods and models for discovering learners' processing of input", in Studies in Second Language Acquisition, 7: 1-14.

Eckerth, J. (2003). Fremdsprachenerwerb in aufgabenbasierten Interaktionen. Tübingen: Narr.

Ellis, R. (1995). "Modified input and the acquisition of word meanings", en. Applied Linguistics, $16,4$.

Ellis, R. (1999a). "Theoretical Perspectives on Interaction and Language Learning", in N, Ellis; S. Fotos (eds.), Learning a second language through interaction. Amsterdam: John Benjamins, 3-31.

Ellis, R. (1999b). "Making the Classroom Acquisition Rich", in N, Ellis \& S. Fotos (eds.), Learning a second language through interaction. Amsterdam: John Benjamins, 211-229.

Ellis, R. (2012). Language teaching research and language pedadogy. Malden, MA: Wiley-Blackwell.

Faerch, C. \& Kasper, G. (1986). "The role of comprehension in second language learning", in Applied Linguistics, 7: 257-274.

Just, M.A. \& Carpenter, P.A. (1992). "A capacity theory of comprehension: Individual differences in working memory". Psychological Review, 99: 122-149.

Krashen, S.D. (1982). Principles and practice in second language acquisition. Oxford: Pergamon.

Lee, J.F. \& VanPatten, B. (1995). Making communicative language teaching happen. NY:McGraw-Hill. 
Long, M. (1991). "Focus on Form: A design feature in language teaching methodology", in K, De Bot (ed.), Foreign Language Research in Cross-Cultural Perspective. Amsterdam: John Benjamins, 39-52.

Long, M. (2015). Second Language Acquisition and Task-Based Language Teaching. Malden, MA: Wiley Blackwell.

Philp, J. \& Tognini, R. (2009). "Language acquisition in foreign language contexts and the differential benefits of interaction", in IRAL 47, 3-4: 245-266.

Rotter, D. (2015). Der Focus-on-Form-Ansatz in der Sprachförderung. Eine empirische Untersuchung der Lehrer-Lerner-Interaktion im DaZ-Grundschulkontext. Waxmann: Münster.

Schmidt, R.W. (1990). "The role of consciousness in second language learning", in Applied Linguistics, 11: 129-158.

Schmidt, R.W. (2001). "Attention”, in P., Robinson (ed.), Cognition and second language instruction. Cambridge: Cambridge University Press, 3-32.

Skehan, P. (1996). "A framework for the implementation of task-based instruction", in Applied Linguistics, 17, 1: 38-62.

Skehan, P. (2003). "Task-based Instruction", in Language Teaching 36, 1: 1-14.

Swain, M. (1991). "French immersion and its off-schoots: Getting two for one", in B.F., Freed (ed.), Foreign language acquisition research and the classroom. Lexington, MA: DC.Heath, 91-103.

Swain, M. (1998). "Focus on form through conscious reflection", in C.J., Doughty \& J. Williams (eds.), Focus-on-form in classroom second language acquisition. Cambridge: Cambridge University Press, 64-81.

Terrel, T. (1991). "The role of grammar instruction in the communicative approach". The Modern Language Journal, 70: 213-227.

VanPatten, B. (1993). "Grammar teaching for the acquisition-rich classroom", in Foreign Language Annals, 26: 435-450.

VanPatten, B. (1996). Input processing and grammar instruction: Theory and research. Norwood: Ablex.

VanPatten, B. (2000). "Processing instruction as form-meaning connections: Issues in theory and research", in J.F. Lee \& A. Valdman (eds.), Form and meaning in language teaching. Boston: Heinle\&Heinle, 43-68.

VanPatten, B. (2002). "Processing Instruction: An update", in Language Learning, 52: 755-803.

VanPatten, B. (2010). "Input Processing in SLA", in B., VanPatten (ed.). Processing Instruction. Theory, Research, and Commentary. NY: Routledge, 5-31.

Wong, W. (2010). "The Nature of Processing Instruction", in in B., VanPatten (ed.). Processing Instruction. Theory, Research, and Commentary. NY: Routledge, 33-63. 